\title{
Erratum: Structure and Dynamics of Loosely Cross-Linked Ionic Microgel Dispersions in the Fluid Regime [Phys. Rev. Lett. 109, 048302 (2012)]
}

P. Holmqvist, P. S. Mohanty, G. Nägele, P. Schurtenberger, and M. Heinen

(Received 23 September 2016; published 20 October 2016)

DOI: 10.1103/PhysRevLett.117.179901

Erroneous values for the concentrations in molar units were reported in parts of the text and the figure captions. The following values should be used instead:

Page 2

Column 1, row 13: $n_{p}=0.022 \mu \mathrm{M}$ should be $n_{p}=0.0167 \mu \mathrm{M}$.

Column 1, row 37 (last row): $n_{p}=0.022 \mu \mathrm{M}$ should be $n_{p}=0.0167 \mu \mathrm{M}$.

Figure 1, figure text: $n_{p}=0.022 \mu \mathrm{M}$ should be $n_{p}=0.0167 \mu \mathrm{M}$.

Figures 2(d), 2(e), and 2(f): $n_{p}=0.022 \mu \mathrm{M}$ should be $n_{p}=0.0167 \mu \mathrm{M}$.

Figure 2, figure text: $n_{p}=0.022 \mu \mathrm{M}$ should be $n_{p}=0.0167 \mu \mathrm{M}$.

Page 3

Figures 3(d), 3(e), and 3(f): $n_{p}=0.022 \mu \mathrm{M}$ should be $n_{p}=0.0167 \mu \mathrm{M}$.

Figure 3, figure text: $n_{p}=0.022 \mu \mathrm{M}$ should be $n_{p}=0.0167 \mu \mathrm{M}$.

Page 4

Figure 4(b): The concentrations should be (i) black rectangle, $n_{p}=0.0053 \mu \mathrm{M}$; (ii) red circle, $n_{p}=0.0060 \mu \mathrm{M}$; (iii) green triangle, $n_{p}=0.0100 \mu \mathrm{M}$; (iv) blue triangle, $n_{p}=0.0167 \mu \mathrm{M}$; (v) blue diamond, $n_{p}=0.0690 \mu \mathrm{M}$.

The interval stated on page 2, column 1, row 27 is correct.

The results presented and conclusions drawn in the Letter are unaffected, since for the calculation of the static structure factor and wave number-dependent diffusion function the correct values of the concentrations were used. 